\title{
Effectiveness of canakinumab treatment in Schnitzler's syndrome: a multi-center randomized placebo-controlled study
}

\author{
K Krause $^{1 *}$, A Tsianakas ${ }^{2}$, N Wagner ${ }^{3}$, J Fischer ${ }^{4}$, K Weller $^{1}$, M Metz $^{1}$, M Maurer $^{1}$ \\ From 8th International Congress of Familial Mediterranean Fever and Systemic Autoinflammatory Diseases \\ Dresden, Germany. 30 September - 3 October 2015
}

\section{Background}

Schnitzler's syndrome (SchS) is an adult-onset autoinflammatory disease characterized by urticarial exanthema and monoclonal gammopathy in combination with episodes of fever, arthralgia, fatigue, and bone and muscle pain. AntiIL-1 targeting therapies in small patient numbers showed to be effective in reducing the clinical symptoms of SchS.

\section{Methods}

The current placebo-controlled multi-center study was designed to assess the effects of the anti-IL-1ß monoclonal antibody canakinumab (CAN) on the clinical signs and symptoms of SchS. We randomly assigned 20 patients with active disease to receive CAN $150 \mathrm{mg}$ or placebo s.c. injections (day 0). Following the evaluation of treatment responses on day 7 the study was continued by a 16-week open label phase with CAN injections upon confirmed relapse of clinical symptoms. Efficacy was determined by changes in the physician's global assessment (PGA; range 0-20), a combined symptom score which includes 5 key symptoms of SchS (urticarial rash, fever, fatigue, myalgia and arthralgia/bone pain), measurement of the inflammation markers $\mathrm{C}$-reactive protein (CRP) and serum amyloid A (SAA) as well as changes in quality of life assessment (DLQI, SF-36).

\section{Results}

CAN was highly effective in reducing median PGA total scores (14.0 to 2.0) as compared to placebo treatment (15.0 to 13.0) within 7 days after first administration (changes between treatment groups $\mathrm{p}<0.0001$ ). Median CRP reduced from $9.3 \mathrm{mg} / \mathrm{dL}$ at baseline to $0.6 \mathrm{mg} / \mathrm{dL}$ at

${ }^{1}$ Charité-Universitätsmedizin Berlin, Dermatology, Berlin, Germany Full list of author information is available at the end of the article day 7 in the CAN group vs. increase from $3.0 \mathrm{mg} / \mathrm{dL}$ to $5.0 \mathrm{mg} / \mathrm{dL}$ for the placebo group. Similarly, median SAA levels reduced from $428 \mathrm{mg} / \mathrm{L}$ to $13 \mathrm{mg} / \mathrm{L}$ for the CAN group vs. increase from $160 \mathrm{mg} / \mathrm{L}$ to $205 \mathrm{mg} / \mathrm{L}$ for the placebo group. The median changes from baseline to day 7 between treatment groups for CRP $(p=0.002)$ and SAA ( $p=0.032$ ) were significant. In addition, quality of life markedly improved. Changes in both physical component SF-36 scores and in DLQI sum scores were significantly greater $(\mathrm{p}<0.0001)$ in the CAN vs. placebo group. The clinical and laboratory improvements were maintained during the open label phase of the study. Also, all placebo-treated patients responded well to CAN therapy during the open-label phase. Adverse events were manageable and included respiratory tract infections, gastrointestinal symptoms and hypertension.

\section{Conclusion}

In this placebo-controlled study, CAN s.c. injections significantly improved the clinical signs and symptoms of SchS, reduced inflammation markers, and enhanced quality of life. CAN treatment may be considered a promising therapeutic option in these patients.

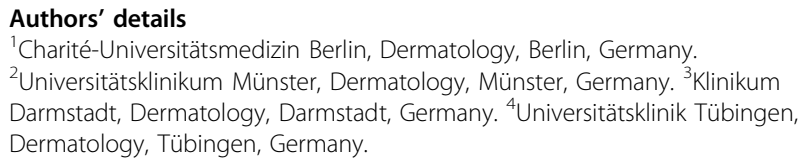

Published: 28 September 2015

doi:10.1186/1546-0096-13-S1-066

Cite this article as: Krause et al.: Effectiveness of canakinumab

treatment in Schnitzler's syndrome: a multi-center randomized placebocontrolled study. Pediatric Rheumatology 2015 13(Suppl 1):O66. 